OPEN ACCESS

Edited by: Detlev Boison, Legacy Health, United States

Reviewed by: Julie A. Saugstad, Oregon Health \& Science University, United States Chuang Wang Ningbo University, China

${ }^{*}$ Correspondence: Young-Kook Kim ykk@jnu.ac.kr

Received: 10 April 2017 Accepted: 03 July 2017 Published: 18 July 2017

Citation:

Song $J$ and Kim Y-K (2017) Identification of the Role of miR-142-5p in Alzheimer's Disease by Comparative Bioinformatics and Cellular Analysis.

Front. Mol. Neurosci. 10:227. doi: 10.3389/fnmol.2017.00227

\section{Identification of the Role of miR-142-5p in Alzheimer's Disease by Comparative Bioinformatics and Cellular Analysis}

\author{
Juhyun Song ${ }^{1}$ and Young-Kook Kim ${ }^{1,2 *}$ \\ ${ }^{1}$ Department of Biomedical Sciences, Center for Creative Biomedical Scientists at Chonnam National University, Gwangju, \\ South Korea, ${ }^{2}$ Department of Biochemistry, Chonnam National University Medical School, Jeollanam-do, South Korea
}

Alzheimer's disease $(A D)$ is the most common neurodegenerative disease characterized by the formation of amyloid beta $(A \beta)$ or tau protein aggregates, the hallmark of cognitive decline. MicroRNAs (miRNAs) have emerged as critical factors in neurogenesis and synaptic functions in the central nervous system (CNS). Recent studies have reported alterations in miRNA expression in patients with AD. However, miRNAs associated with $A D$ varied with patient groups or experimental models, suggesting the need for a comparative study to identify miRNAs commonly dysregulated in diverse $A D$ models. Here, we investigated the miRNAs that show dysregulated expression in two different human $A D$ groups and mouse and cellular $A D$ models. After selection of commonly dysregulated miRNAs in these groups, we investigated the pathophysiological significance of miR-142-5p in SH-SY5Y neuronal cells. We found that miR-142-5p was increased upon treatment with $A \beta$ peptide 1-42 $\left(A \beta_{42}\right)$. Inhibition of miR-142-5p rescued the $A \beta_{42}$-mediated synaptic dysfunctions, as indicated by the expression of postsynaptic density protein 95 (PSD-95). Among genes with decreased expression in $\mathrm{A} \beta_{42}$-treated $\mathrm{SH}-\mathrm{SY} 5 \mathrm{Y}$ cells, the predicted miR-142-5p target genes were significantly related with neuronal function and synapse plasticity. These findings suggest that dysregulation in miR-142-5p expression contributes the pathogenesis of $A D$ by triggering synaptic dysfunction associated with $A \beta_{42}$-mediated pathophysiology.

Keywords: miR-142-5p, Alzheimer's disease (AD), synaptic plasticity, SH-SY5Y cells, postsynaptic density protein 95 (PSD-95)

\section{INTRODUCTION}

MicroRNAs (miRNAs) are non-coding RNA molecules that comprise approximately 22 nucleotides and suppress the translation of mRNAs by binding to their $3^{\prime}$-untranslated region (UTR; Davis et al., 2015; Kim, 2015; Cao et al., 2016; Reddy et al., 2017). As miRNAs regulate a variety of cellular processes, modulation of miRNA expression is considered a promising therapeutic approach for the diagnosis and treatment of diverse central nervous system (CNS) diseases (Gascon and Gao, 2012; Grasso et al., 2014; Qiu et al., 2015; Martinez and Peplow, 2016). Several studies demonstrated that over 70\% of identified miRNAs are found in the brain (Shao et al., 2010). miRNAs are shown to control a variety of cellular mechanisms such as development, neuron survival, synaptic plasticity and secretion of neurotransmitter in the CNS (Cohen et al., 2011). 
Aberrations in the expression of miRNAs in the CNS contribute to neurodegenerative disorders, including Alzheimer's, Parkinson's and Huntington's diseases (Cogswell et al., 2008; Saba and Schratt, 2010; Gascon and Gao, 2012; Salta and De Strooper, 2012; Grasso et al., 2014). Alzheimer's disease $(\mathrm{AD})$ is the most common neurodegenerative disease characterized by the formation of amyloid plaques, which are associated with neuronal loss and cognitive decline (O'Brien and Wong, 2011; Reddy et al., 2012). Studies have reported that the cognitive deficit in $\mathrm{AD}$ results from impaired synaptic transmission and plasticity by aberrant amyloid beta peptide 1-42 (A $\beta_{42}$; Jo et al., 2011; Sheng et al., 2012). Clinical studies have reported an alteration in the miRNA expression in $\mathrm{AD}$ patients compared to normal subjects, which contributes to disease progression (Cogswell et al., 2008; Tan et al., 2014; Denk et al., 2015). Although these studies identified several miRNA candidates as biomarkers in $\mathrm{AD}$, the detailed functional studies on miRNAs that contribute to the pathogenesis of $\mathrm{AD}$, such as synaptic dysfunctions and cognitive decline, are largely unknown.

In the present study, we investigated miRNAs that show dysregulated expression in $\mathrm{AD}$. We evaluated alterations in miRNA expressions in both the human and the mouse $\mathrm{AD}$ brain through comparative bioinformatics analysis. We selected the target candidate miRNA in the $A D$ brain and analyzed its role in $\mathrm{A} \beta_{42}$-treated SH-SY5Y neuronal cells in vitro. Through the analysis of target genes of miRNA, we suggest the importance of miRNA in $\mathrm{AD}$ pathogenesis. Thus, we highlight that modulation of a specific miRNA in the $\mathrm{AD}$ brain is necessary to improve $\mathrm{AD}$ pathogenesis.

\section{MATERIALS AND METHODS}

\section{Analysis of miRNA Expression from Sequencing Data}

We identified miRNAs with altered expression in AD models using raw data from small RNA sequencing obtained from Gene Expression Omnibus (GEO; GSE48552, GSE63501 and GSE55589) ${ }^{1}$. After deletion of the adaptor sequences, sequences with low quality were eliminated using FASTX-Toolkit ${ }^{2}$ and the remaining sequences were aligned to the human reference genome (GRCh38) by Bowtie2 (Langmead and Salzberg, 2012). Only the reads perfectly aligned to the human genome were selected. Genomic coordinates of miRNAs (obtained from http://www.mirbase.org/) modified with manual curation were used to calculate the miRNA counts (Kim et al., 2016). Using an in-house Perl script, we counted the sequencing reads corresponding to miRNA loci and normalized them by scaling whole read counts as one million (RPM, reads per million). miRNAs with average RPM values higher than 5 in each dataset were used for further analyses (Figure 1A).

\footnotetext{
${ }^{1}$ https://www.ncbi.nlm.nih.gov/geo/

${ }^{2}$ http://hannonlab.cshl.edu/fastx_toolkit/
}

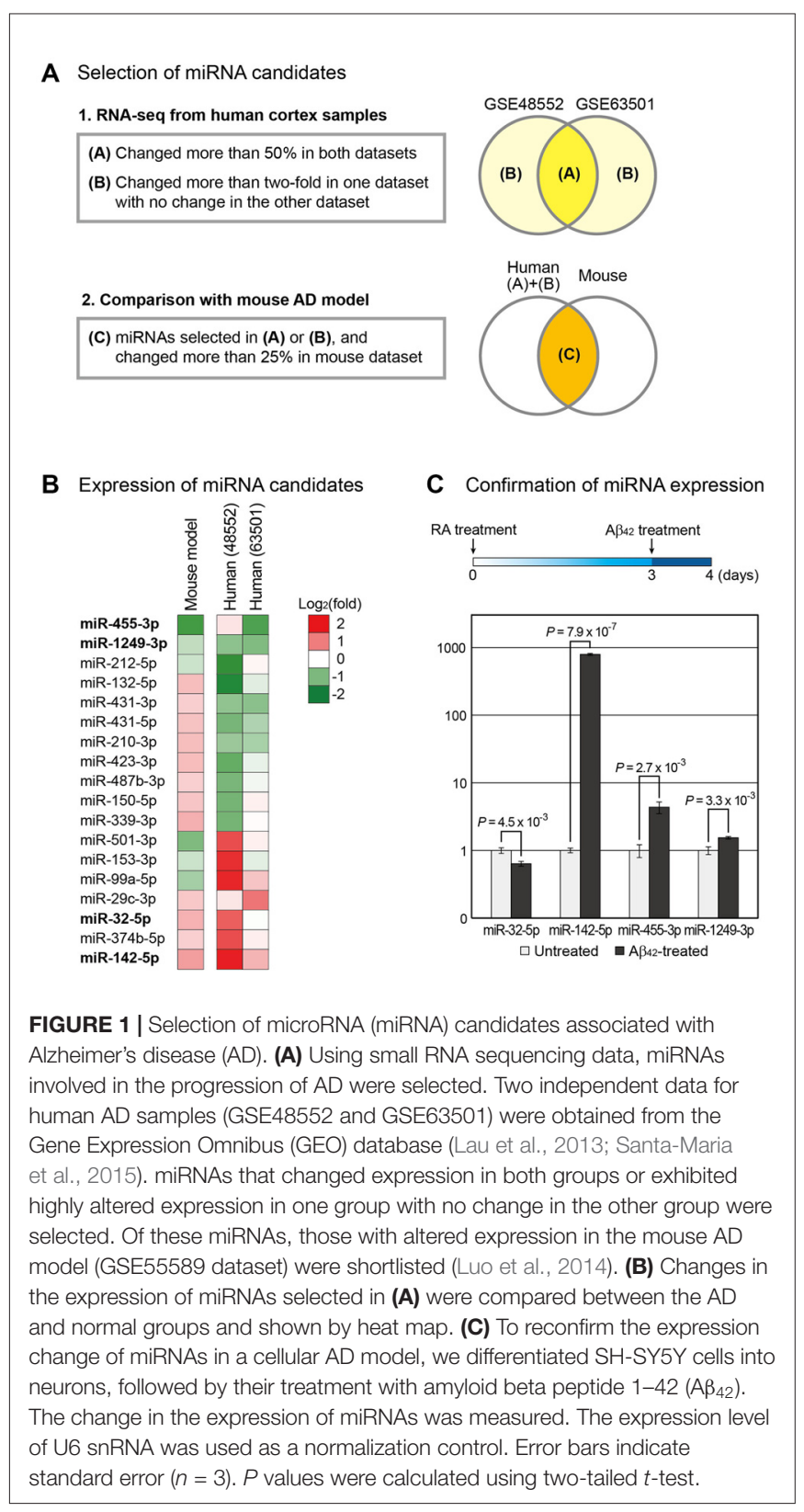

\section{Cell Culture and Transfection}

The SH-SY5Y neuroblastoma cell line was used in this study. SH-SY5Y cells are capable of differentiating into neuron-like cells in presence of retinoic acid (RA; Encinas et al., 1999; Xun et al., 2012; Shipley et al., 2016). Undifferentiated SH-SY5Y cells were cultured in Dulbecco's Modified Eagle's Medium (DMEM) supplemented with $10 \%$ fetal bovine serum (FBS; Gibco, Grand Island, NY, USA) and $100 \mu \mathrm{g} / \mathrm{mL}$ penicillinstreptomycin (Gibco, Grand Island, NY, USA) at $2 \times 10^{5}$ cells/mL. After 2 days, the medium was replaced with DMEM supplemented with $10 \%$ FBS and $5 \mu \mathrm{M}$ RA for neuronal differentiation. SH-SY5Y cells were cultured in a humidified atmosphere of $5 \% \mathrm{CO}_{2}$ at $37^{\circ} \mathrm{C}$. Synthetic miR-142-5p and control inhibitor were purchased from Ambion (Ambion, 
Austin, TX, USA). This inhibitor specifically binds to and blocks the function of endogenous miRNAs. SH-SY5Y cells were cultured for 3 days, followed by inhibitor treatment using Lipofectamine 3000 reagent (Thermo Fisher Scientific, Carlsbad, CA, USA) and incubation for one and a half days. One day before sampling, cells were treated with $10 \mu \mathrm{M}$ $\mathrm{A} \beta_{42}$.

\section{$A \beta_{42}$ Oligomer Preparation}

Oligomeric $A \beta_{42}$ was prepared as previously described (An et al., 2013). Briefly, $1 \mathrm{mg}$ of synthetic $A \beta_{42}$ peptide (American Peptide,

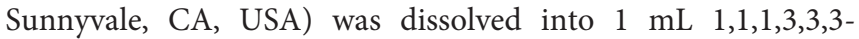
hexafluoro-2-propanol (HFIP; Sigma Aldrich, St. Louis, MO, USA). The solution was dried under a nitrogen stream and resuspended in $100 \%$ HFIP at a concentration of $1 \mathrm{mg} / \mathrm{mL}$. The solution was sonicated in a bath sonicator for $5 \mathrm{~min}$ and dried under nitrogen stream. The solution was re-dissolved in $1 \mathrm{~mL}$ HFIP and dried for $2 \mathrm{~h}$. The film was resuspended in $200 \mu \mathrm{L}$ dimethyl sulfoxide (DMSO; Sigma Aldrich, St. Louis, MO, USA) to obtain a $1 \mathrm{mM} \mathrm{A} \beta_{42}$ stock solution. For $A \beta_{42}$ treatment, $10 \mu \mathrm{L}$ of $1 \mathrm{mM} \mathrm{A} \beta_{42}$ was diluted with $1 \mathrm{~mL}$ DMEM media (final concentration, $10 \mu \mathrm{M}$ ) and incubated for $12 \mathrm{~h}$ at $37^{\circ} \mathrm{C}$.

\section{Measurement of miRNA Levels}

After removing culture media, SH-SY5Y cells were collected using cell scraper (Sigma-Aldrich, St. Louis, MO, USA). After centrifuging at $12,000 \mathrm{~g}$ for $3 \mathrm{~min}$ at $4^{\circ} \mathrm{C}$, we used only cell pellets for RNA isolation. Total RNA in SH-SY5Y cells was extracted using TRIzol reagent (Thermo Fisher Scientific, Carlsbad, CA, USA). The concentration of RNA was measured by NanoDrop spectrophotometer (ND-1000, NanoDrop technology). Quantitative analysis of miR-325p, miR-142-5p, miR-455-3p and miR-1249-3p expression was performed with reverse transcription polymerase chain reaction (RT-PCR) using the TaqMan MicroRNA Reverse Transcription kit (Applied Biosystems, Waltham, MA, USA) and $10 \mathrm{ng}$ of total RNA. PCR reactions were performed following the manufacturer's guideline to quantitate the level of miR-32-5p, miR-142-5p, miR-455-3p and miR-1249-3p using TaqMan Universal PCR Master Mix, No Amp Erase UNG (Applied Biosystems, Waltham, MA, USA), and TaqMan MicroRNA assay (Applied Biosystems, Waltham, MA, USA). PCR amplification was conducted in Takara Real Time PCR (Takara, Shiga, Japan). Levels of miRNAs were presented as relative quantities (RQ) normalized to the level of U6 snRNA.

\section{Western Blot Analysis}

For western blot analysis, SH-SY5Y cells were cultured in $100 \mathrm{~mm}$ plates. After treating the cells with each chemical, we harvested them using cell scraper (Sigma-Aldrich, St. Louis, MO, USA). After centrifuging at 12,000 $g$ for $3 \mathrm{~min}$, the supernatants were removed. The cell pellets were rinsed with phosphate-buffered saline (PBS) and collected by centrifugation again. SH-SY5Y cell pellets were lysed with ice-cold radioimmunoprecipitation assay
(RIPA) buffer (Sigma-Aldrich, St. Louis, MO, USA). Cell lysates were centrifuged at $12,000 \mathrm{~g}$ for $30 \mathrm{~min}$ at $4^{\circ} \mathrm{C}$ to produce whole-cell extracts. Proteins $(30 \mu \mathrm{g})$ were separated on a $10 \%$ sodium dodecyl sulfate (SDS)-polyacrylamide gel and transferred onto a polyvinylidene difluoride membrane. The membrane was blocked with skim milk prepared in TBS-T (20 nM Tris $\mathrm{pH} 7.2,150 \mathrm{mM}$ sodium chloride $[\mathrm{NaCl}], 0.1 \%$ Tween 20) for $90 \mathrm{~min}$ at room temperature, followed by incubation with primary antibody against amyloid precursor protein (APP; 1:1000; Abcam, Cambridge, MA, USA), A $\beta_{42}$ (1:1000; Abcam, Cambridge, MA, USA) or $\beta$-actin (1:1000; Millipore, Billerica, MA, USA) for $18 \mathrm{~h}$ at $4^{\circ} \mathrm{C}$. The membrane was then probed with a secondary antibody (Abcam, Cambridge, MA, USA) for $90 \mathrm{~min}$ at room temperature and visualized using enhanced chemiluminescence (ECL) solution (Millipore, Billerica, MA, USA).

\section{Immunocytochemistry}

SH-SY5Y cells were washed thrice with PBS and fixed for 30 min with $4 \%$ paraformaldehyde solution. Cells were incubated with anti-rabbit postsynaptic density protein 95 (PSD-95; 1:500; Cell Signaling, Danvers, MA, USA) antibody for $18 \mathrm{~h}$ at $4^{\circ} \mathrm{C}$. Following incubation, cells were washed twice with PBS and incubated with a secondary antibody for $90 \mathrm{~min}$ at room temperature. Cells were counterstained with $1 \mu \mathrm{g} / \mathrm{mL}$ of 4',6-diamidino-2-phenylindole (DAPI, 1:100; Thermo Fisher Scientific, Carlsbad, CA, USA) for $15 \mathrm{~min}$ at room temperature. Images were obtained with a confocal microscope (Carl Zeiss, Thornwood, NY, USA) and the intensity of PSD-95 signal was measured using ImageJ software.

\section{Measurement of mRNA Levels}

The amount of PSD-95 mRNA in SH-SY5Y cells was evaluated with quantitative real-time PCR. Total cellular RNA was extracted from SH-SY5Y cells using TRIzol reagent (Thermo Fisher Scientific, Carlsbad, CA, USA) and complementary DNA (cDNA) was synthesized using AccuPower Rocketscript Cycle RT Premix (Bioneer, Daejeon, Korea). The cDNA was mixed with SYBR premix EX-Taq RT-PCR Kit (Takara, Shiga, Japan) and specific primers in a total reaction volume of $20 \mu \mathrm{L}$. PCR was performed using the following primers: PSD-95 forward: 5'-TCGGTGACGACCCATCCAT-3', PSD-95 reverse: 5'-GCACGTCCACTTCATTTACAAAC-3'; glyceraldehyde 3-phosphate dehydrogenase (GAPDH) forward: 5'-ACAACTTTGGTATCGTGGAAGG-3', GAPDH reverse: $5^{\prime}$-GCCATCACGCCACAGTTTC-3'. GAPDH was used as an internal control. The $\Delta \mathrm{Ct}$ values from $\mathrm{A} \beta_{42}$-treated $\mathrm{SH}-\mathrm{SY} 5 \mathrm{Y}$ cells were compared with those from untreated SH-SY5Y cells.

\section{Analysis of miRNA Targets}

We used the predicted target lists from TargetScan database ${ }^{3}$ to identify the target mRNAs of miR-142-5p (Agarwal et al., 2015). Only the transcripts with conserved targets sites corresponding

\footnotetext{
${ }^{3}$ http://www.targetscan.org
} 
to 947 genes were used. As the expression of miR-142-5p is greatly increased in $A \beta_{42}$-treated cells (Figure 1), we selected mRNAs that exhibited decreased expression under same condition, using the microarray data for $\mathrm{A} \beta_{42}$-treated SH-SY5Y cells (GSE23000; Gatta et al., 2011). mRNAs from $\mathrm{A} \beta_{42}$-treated cells with expression below 0.8 -fold compared to those from untreated cells were selected. These mRNAs were intersected with the list of predicted target genes of miR-142-5p. Finally, 102 genes were selected and subjected to gene ontology (GO) analysis at Molecular Signatures Database (Subramanian et al., 2005) ${ }^{4}$. The top 10 most significant GO terms were selected.

\section{RESULTS}

\section{Selection of miRNA Candidates Associated with AD}

miRNAs commonly dysregulated in the brain of $\mathrm{AD}$ patients and mouse model were identified using the high-throughput sequencing data from GEO. GSE48552 and GSE63501 datasets were selected to obtain small RNA sequencing data from human cortex samples. The GSE48552 dataset was obtained from the analysis of the expression of small RNAs from six early-stage (control) and six late-stage $\mathrm{AD}$ patients (Lau et al., 2013). The GSE63501 dataset contained the small RNA sequencing data for brains from seven control and six AD patients (Santa-Maria et al., 2015). Using these two datasets, we selected miRNAs with altered expression in both datasets (more than 50\%) or considerable change (more than two-fold) in either dataset as candidates involved in the pathogenesis of $\mathrm{AD}$ (Figure $\mathbf{1 A}$ and Supplementary Table S1).

We included the small RNA expression data from the mouse model of $\mathrm{AD}$ owing to the possible high variation in human data. We used GSE55589 dataset, which profiled small RNAs from the cortex of APP/PSEN1 $\triangle \mathrm{E} 9$ transgenic mouse and its sibling wild-type pair (Luo et al., 2014). This mouse model expresses mutant forms of APP and presenilin 1 (PSEN1) in neurons; both these proteins are associated with earlyonset $\mathrm{AD}$ in humans. We compared the miRNA expression levels in this dataset to those selected from human datasets and selected only the miRNAs that displayed more than 25\% change in mice (Figure 1A and Supplementary Table S1). Finally, we selected 18 miRNAs from these analyses (Figure 1B).

From the selected list, we chose miRNAs that showed either increased or decreased expression in both humans and mice. Moreover, candidates with other paralogous miRNA in the human genome were excluded to avoid complications in the analysis. Thus, we shortlisted four miRNAs-miR-32-5p, miR142-5p, miR-455-3p and miR-1249-3p.

We reconfirmed the expression change of these miRNAs using the cell-based model for AD. A human neuroblastoma cell line SH-SY5Y that differentiates into neuronal phenotype

${ }^{4} \mathrm{http}: / /$ software.broadinstitute.org/gsea/msigdb on treatment with RA was used (Encinas et al., 1999; Xun et al., 2012; Shipley et al., 2016). After incubation with RA for 3 days, cells were treated with $A \beta_{42}$. Total RNA was extracted the following day and the miRNA level was evaluated by quantitative PCR. We found that miR-142-5p was significantly increased in $A \beta_{42}$-treated cells compared to control, consistent with the brain sample data from both humans and mice (Figure 1C and Supplementary Figure S1A). As a control for $A \beta_{42}$ treatment, we measured the protein level of APP and found that its level evidently increased in $A \beta_{42}$-treated cells (Supplementary Figure $\mathrm{S} 1 \mathrm{~B})$. We selected miR-142-5p as the final candidate involved in the pathogenesis of $\mathrm{AD}$.

\section{miR-142-5p Suppression Inhibits the Loss of PSD-95 in SH-SY5Y Neuronal Cells Treated with $\mathbf{A} \beta_{42}$}

Given the increase in miR-142-5p expression during the pathogenesis of $\mathrm{AD}$, we hypothesized that miR-142-5p suppression may alleviate the $\mathrm{AD}$ phenotype. We pretreated differentiated SH-SY5Y cells with miR-142-5p inhibitor, followed by treatment with $A \beta_{42}$ (Figure $2 \mathrm{~A}$ and Supplementary Figure S2). The effect of miR-142-5p inhibition in $A \beta_{42^{-}}$ treated SH-SY5Y cells was investigated with the evaluation of PSD-95 expression. A loss of synaptic integrity leads to cognitive impairment in the $\mathrm{AD}$ brain (Coleman and Yao, 2003; Gylys et al., 2004), and PSD-95 as a neuronal scaffolding protein (Niethammer et al., 1996) is known to influence synapse maturation and regulate synaptic plasticity in the neuronal network (Kennedy, 2000; Elias et al., 2006). Several studies demonstrated that the level of PSD-95 was considerably reduced in the $\mathrm{AD}$ brain (Gylys et al., 2004; Love et al., 2006). Immunocytochemistry experiment revealed that the intensity of PSD-95 protein in SH-SY5Y cells was reduced after $\mathrm{A} \beta_{42}$ treatment as expected (Figures $\mathbf{2 B}, \mathbf{C}$; Leuba et al., 2008; Singh et al., 2017). However, pretreatment of SH-SY5Y cells with miR-142-5p inhibitor compromised the reduction in PSD-95 after $\mathrm{A} \beta_{42}$ treatment. This result was verified with the measurement of PSD-95 mRNA level (Figure 2D). Pretreatment with miR-142-5p inhibitor attenuated the decrease of PSD-95 mRNA expression after treatment with $A \beta_{42}$. Taken together, our results show that the inhibition of miR-142-5p prevented the $A \beta_{42}$-induced loss of PSD-95 in SH-SY5Y cells.

\section{Target Analysis of miR-142-5p and Its Functional Implication}

Our study shows that miR-142-5p plays a role in neuronal process. To identify target genes of miR-142-5p, we used the microarray data and measured global mRNA level changes after treatment of SH-SY5Y cells with $A \beta_{42}$ (Gatta et al., 2011). Because miR-142-5p expression is highly increased by $A \beta_{42}$ treatment, we selected only the mRNAs with decreased expression following $A \beta_{42}$ treatment. In comparison to untreated controls, cells treated with $\mathrm{A} \beta_{42}$ showed a decrease below 0.8 -fold in the expression of 3021 mRNAs (Figure 3A). As miRNAs regulate the expression of mRNAs by binding to the 


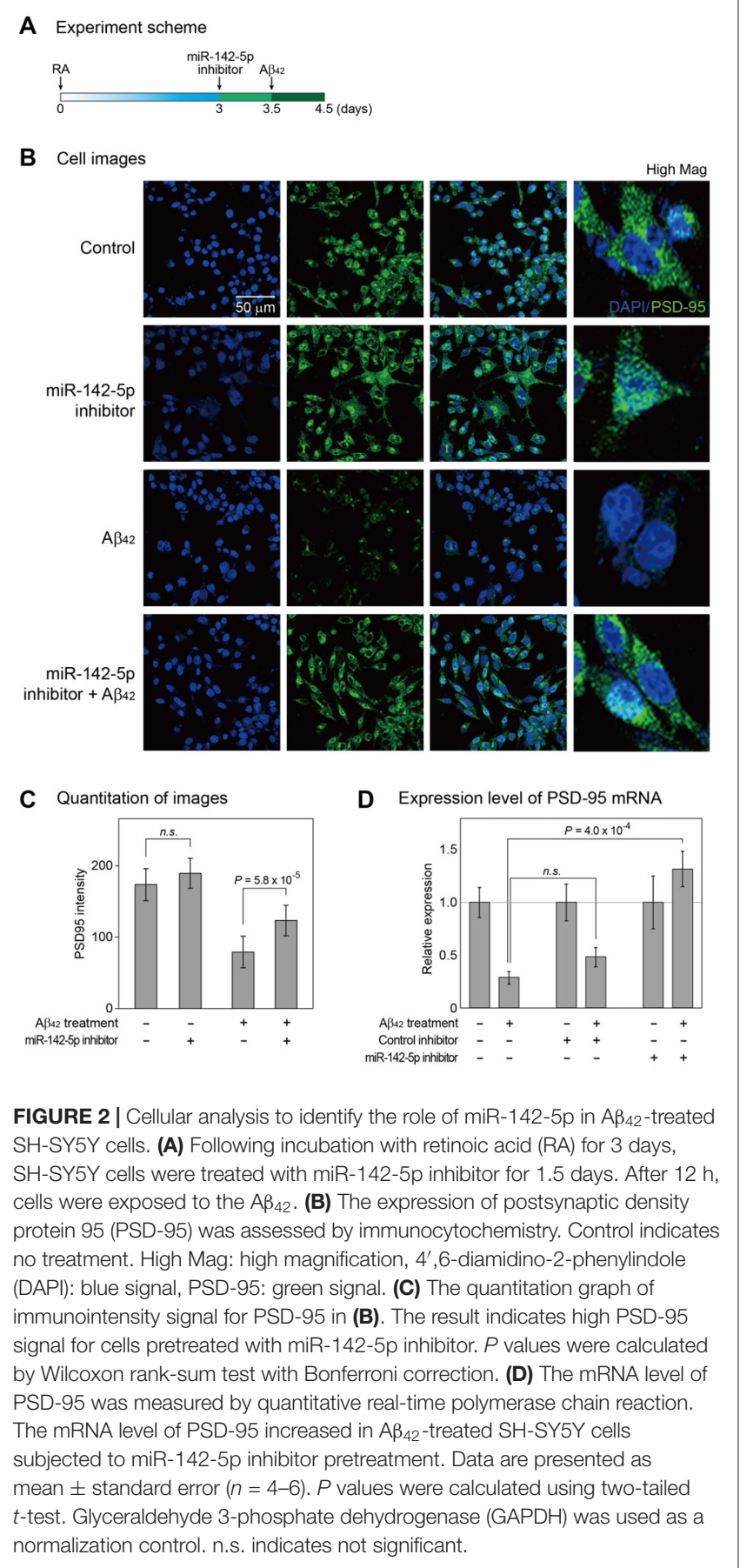

sequence-specific target sites on mRNAs, we intersected these selected genes with the predicted target list of miR-142-5p from TargetScan database (Agarwal et al., 2015). Of 947 genes with conserved target site(s), 102 genes showed decreased expression in $\mathrm{A} \beta_{42}$-treated SH-SY5Y cells (Figure 3A and Supplementary Table S2).

We analyzed the function of these genes by performing gene set analysis. GO analyses identified six neuron-related terms among the top 10 significantly enriched GO terms (Figure 3B).

\section{A Selection of target candidates}

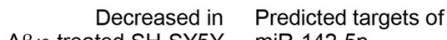

A 342 -treated SH-SY5Y miR-142-5p

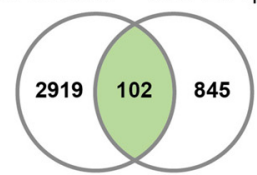

B GO analysis

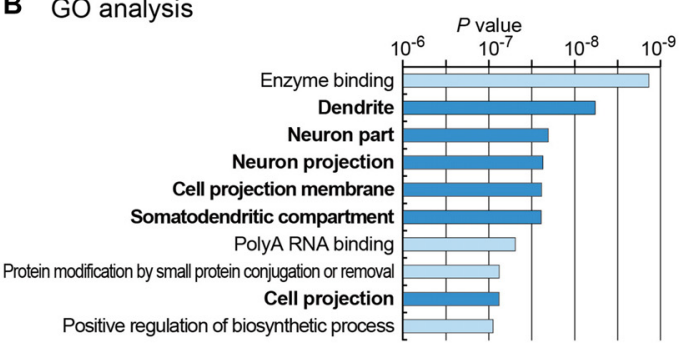

C Target candidates related to neuronal process

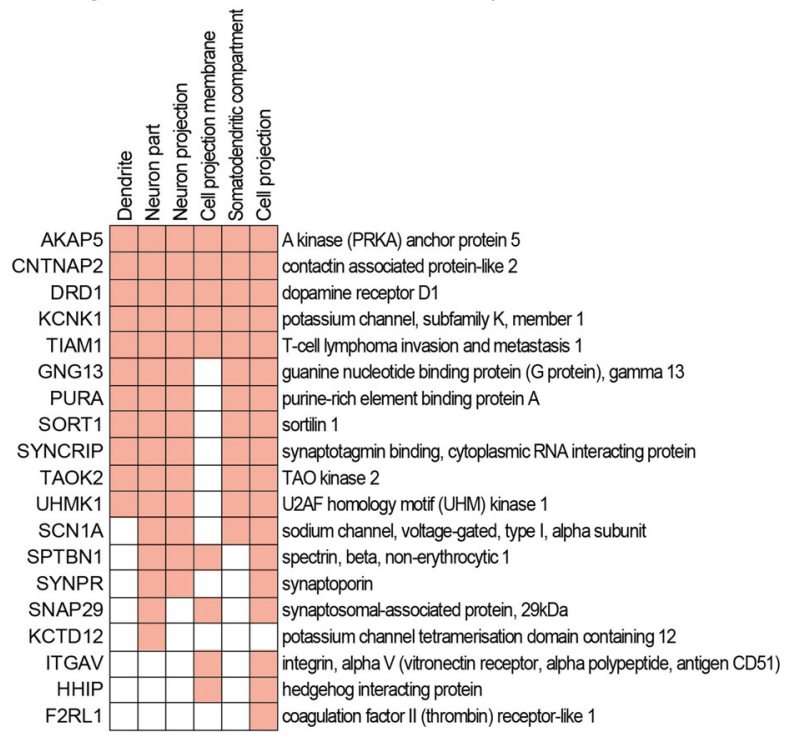

FIGURE 3 | Target analysis of miR-142-5p and its functional implication. (A) For the identification of the target genes of miR-142-5p, we analyzed mRNA expression profile in SH-SY5Y cells after treatment with $A \beta_{42}$ (GSE23000; Gatta et al., 2011). Only genes that exhibited decreased expression after $A \beta_{42}$ treatment were chosen. We filtered these genes based on the existence of conserved target sites of miR-142-5p using predicted target list from TargetScan database (Agarwal et al., 2015). (B) Using the selected gene set in (A), statistically enriched Gene Ontology (GO) terms were identified using Molecular Signatures Database (Subramanian et al., 2005). $P$ values, which were calculated by hypergeometric test for each GO term, were obtained from the database. The terms related with neuronal process are shown with bold letters. (C) Target gene lists of miR-142-5p related with neuronal processes are shown. Among the target genes of miR-142-5p from (A), those genes with neuronal process-related GO terms in (B) were chosen. Genes with corresponding GO terms are shown with filled boxes.

Of 102 selected genes, those with neuron-related GO terms are listed in Figure 3C. GO analysis for the decreased gene group with no target site for miR-142-5p (2919 genes) showed no 
neuron-related term among the top 10 enriched terms (data not shown). This analysis suggests that miR-142-5p plays a specific role in neuronal process during $\mathrm{AD}$ progression by targeting neuron-related genes.

\section{DISCUSSION}

$\mathrm{AD}$ is characterized by progressive impairment of synaptic plasticity, resulting in dysfunction of learning and memory processes (Pozueta et al., 2013). Previous studies identified several miRNAs that regulate the expression of those genes involved in the pathogenesis of AD. Beta-secretase 1 (BACE-1) and APP, which play important roles in AD, were identified as the targets of miR-135a and miR-200b, respectively (Liu et al., 2014). Another study revealed that miR-200c, which suppresses phosphatase and tensin homolog (PTEN), has a protective role in AD pathologies (Wu et al., 2016). These and many other studies showed that miRNAs have important roles in the progression of $\mathrm{AD}$. In the present study, we added a new miRNA to this list, which could be a potential therapeutic target for the treatment of $\mathrm{AD}$. We found that miR-142-5p suppression prevents the reduction of PSD-95 level in $\mathrm{A} \beta_{42^{-}}$ treated SH-SY5Y neuronal cells. In addition, bioinformatics analysis revealed the regulation of genes associated with neuronal maintenance and synaptic plasticity by miR-142-5p. As abnormal synaptic process is an important factor in cognitive dysfunction, our results suggest that miR-142-5p may be a potential target in $\mathrm{AD}$ for the improvement in synaptic signaling.

PSD-95, a major scaffold postsynaptic protein, affects the functional integrity of excitatory synapses (El-Husseini Ael et al., 2002; Blanpied et al., 2008). Previous studies showed a decrease in PSD-95 level in brains of AD patients (Gylys et al., 2004; Proctor et al., 2010), leading to neuronal death and memory deficits (Oakley et al., 2006; Leuba et al., 2008; Proctor et al., 2010). PSD-95 was identified as a target of miR-125-5p (Muddashetty et al., 2011). However, miR-125-5p expression showed no change in human $\mathrm{AD}$ samples and exhibited a slight decrease in the mouse AD model (data not shown) and hence, may not be relevant to the pathogenesis of $\mathrm{AD}$. Although the decrease in PSD-95 expression after $\mathrm{A} \beta_{42}$ treatment was compromised through the inhibition of miR-142-5p, the underlying mechanism is still unclear. When we searched a possible binding site of miR-142-5p in the $3^{\prime}$-UTR of PSD-95, no site was identified from several target search algorithms (data not shown). Supporting this, there is no sequence motif (ACUUUAU) in the $3^{\prime}$-UTR of

\section{REFERENCES}

Agarwal, V., Bell, G. W., Nam, J.-W., and Bartel, D. P. (2015). Predicting effective microRNA target sites in mammalian mRNAs. Elife 4:e05005. doi: 10.7554/eLife.05005

An, K., Klyubin, I., Kim, Y., Jung, J. H., Mably, A. J., O’Dowd, S. T., et al. (2013). Exosomes neutralize synaptic-plasticity-disrupting activity of $\mathrm{A} \beta$ assemblies in vivo. Mol. Brain 6:47. doi: 10.1186/1756-6606-6-47
PSD-95 which is complementary to the seed sequence of miR142-5p (AUAAAGU). This suggests that PSD-95 would be regulated by miR-142-5p indirectly. One possibility is that the suppression of miR-142-5p target genes may have decreased the expression of PSD-95. Several target genes shown in Figure 3C, including those for A-kinase anchor protein (AKAP5) and dopamine receptor D1 (DRD1), are known to interact with PSD-95. In neurons, AKAP5 directly interacts with PSD-95 at postsynaptic density, leading to the endocytosis of synaptic AMPA receptors (Colledge et al., 2000; Bhattacharyya et al., 2009). DRD1 interacts with the N-terminus of PSD-95 and maintains the balance of dopamine receptor-glutamatergic scaffold interaction (Zhang et al., 2007). Thus, miR-142-5p may affect PSD-95 indirectly by regulating the expression of other neuron-related genes. This necessitates further studies to gain insight in the detail mechanism of the effect of miR-142-5p during the pathogenesis of AD.

There need to be additional studies to use miR-142-5p as a therapeutic target of $\mathrm{AD}$. One of the key experiments is to test whether the inhibition of miR-142-5p expression in the brain of animal AD model could restore the loss of PSD-95 and subsequently prevent neuronal loss and memory decline. Thus, the establishment of a proper method to manipulate the level of miR-142-5p would be important to verify the role of this miRNA in the regulation of synaptic plasticity and to alleviate the pathogenesis of $\mathrm{AD}$.

\section{AUTHOR CONTRIBUTIONS}

JS conducted cellular experiments and wrote the preliminary draft. Y-KK performed bioinformatics analysis, revised the manuscript and provided overall supervision for the project.

\section{ACKNOWLEDGMENTS}

This study was supported by the Basic Science Research Program through the National Research Foundation of Korea (NRF), funded by the Ministry of Science, ICT and Future Planning (NRF-2015R1C1A1A02036313).

\section{SUPPLEMENTARY MATERIAL}

The Supplementary Material for this article can be found online at: http://journal.frontiersin.org/article/10.3389/fnmol.20 17.00227/full\#supplementary-material

Bhattacharyya, S., Biou, V., Xu, W., Schlüter, O., and Malenka, R. C. (2009). A critical role for PSD-95/AKAP interactions in endocytosis of synaptic AMPA receptors. Nat. Neurosci. 12, 172-181. doi: 10.1038/nn.2249

Blanpied, T. A., Kerr, J. M., and Ehlers, M. D. (2008). Structural plasticity with preserved topology in the postsynaptic protein network. Proc. Natl. Acad. Sci. U S A 105, 12587-12592. doi: 10.1073/pnas.0711669105

Cao, D.-D., Li, L., and Chan, W.-Y. (2016). MicroRNAs: key regulators in the central nervous system and their implication in 
neurological diseases. Int. J. Mol. Sci. 17:e842. doi: 10.3390/ijms170 60842

Cogswell, J. P., Ward, J., Taylor, I. A., Waters, M., Shi, Y., Cannon, B., et al. (2008). Identification of miRNA changes in Alzheimer's disease brain and CSF yields putative biomarkers and insights into disease pathways. J. Alzheimers Dis. 14, 27-41. doi: 10.3233/jad-2008-14103

Cohen, J. E., Lee, P. R., Chen, S., Li, W., and Fields, R. D. (2011). MicroRNA regulation of homeostatic synaptic plasticity. Proc. Natl. Acad. Sci. U S A 108, 11650-11655. doi: 10.1073/pnas.1017576108

Coleman, P. D., and Yao, P. J. (2003). Synaptic slaughter in Alzheimer's disease. Neurobiol. Aging 24, 1023-1027. doi: 10.1016/j.neurobiolaging.2003.09.001

Colledge, M., Dean, R. A., Scott, G. K., Langeberg, L. K., Huganir, R. L., and Scott, J. D. (2000). Targeting of PKA to glutamate receptors through a MAGUK-AKAP complex. Neuron 27, 107-119. doi: 10.1016/s08966273(00)00013-1

Davis, G. M., Haas, M. A., and Pocock, R. (2015). MicroRNAs: not "fine-tuners" but key regulators of neuronal development and function. Front. Neurol. 6:245. doi: 10.3389/fneur.2015.00245

Denk, J., Boelmans, K., Siegismund, C., Lassner, D., Arlt, S., and Jahn, H. (2015). MicroRNA profiling of CSF reveals potential biomarkers to detect Alzheimer's disease. PLoS One 10:e0126423. doi: 10.1371/journal.pone.0126423

El-Husseini Ael, D., Schnell, E., Dakoji, S., Sweeney, N., Zhou, Q., Prange, O., et al. (2002). Synaptic strength regulated by palmitate cycling on PSD-95. Cell 108, 849-863. doi: 10.1016/s0092-8674(02)00683-9

Elias, G. M., Funke, L., Stein, V., Grant, S. G., Bredt, D. S., and Nicoll, R. A. (2006). Synapse-specific and developmentally regulated targeting of AMPA receptors by a family of MAGUK scaffolding proteins. Neuron 52, 307-320. doi: 10.1016/j.neuron.2007.01.007

Encinas, M., Iglesias, M., Llecha, N., and Comella, J. X. (1999). Extracellularregulated kinases and phosphatidylinositol 3-kinase are involved in brainderived neurotrophic factor-mediated survival and neuritogenesis of the neuroblastoma cell line SH-SY5Y. J. Neurochem. 73, 1409-1421. doi: 10.1046/j. 1471-4159.1999.0731409.x

Gascon, E., and Gao, F. B. (2012). Cause or effect: misregulation of microRNA pathways in neurodegeneration. Front. Neurosci. 6:48. doi: 10.3389/fnins.2012. 00048

Gatta, V., Drago, D., Fincati, K., Valenti, M. T., Dalle Carbonare, L., Sensi, S. L., et al. (2011). Microarray analysis on human neuroblastoma cells exposed to aluminum, $\beta_{1-42}$-amyloid or the $\beta_{1-42}$-amyloid aluminum complex. PLoS One 6:e15965. doi: 10.1371/journal.pone.0015965

Grasso, M., Piscopo, P., Confaloni, A., and Denti, M. A. (2014). Circulating miRNAs as biomarkers for neurodegenerative disorders. Molecules 19, 6891-6910. doi: 10.3390/molecules 19056891

Gylys, K. H., Fein, J. A., Yang, F., Wiley, D. J., Miller, C. A., and Cole, G. M. (2004). Synaptic changes in Alzheimer's disease: increased amyloid$\beta$ and gliosis in surviving terminals is accompanied by decreased PSD-95 fluorescence. Am. J. Pathol. 165, 1809-1817. doi: 10.1016/S0002-9440(10) 63436-0

Jo, J., Whitcomb, D. J., Olsen, K. M., Kerrigan, T. L., Lo, S. C., Bru-Mercier, G., et al. (2011). A $\beta_{1-42}$ inhibition of LTP is mediated by a signaling pathway involving

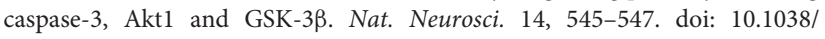
nn. 2785

Kennedy, M. B. (2000). Signal-processing machines at the postsynaptic density. Science 290, 750-754. doi: 10.1126/science.290.5492.750

Kim, Y. K. (2015). Extracellular microRNAs as biomarkers in human disease. Chonnam Med. J. 51, 51-57. doi: 10.4068/cmj.2015.51.2.51

Kim, Y. K., Kim, B., and Kim, V. N. (2016). Re-evaluation of the roles of DROSHA, Export in 5, and DICER in microRNA biogenesis. Proc. Natl. Acad. Sci. U S A 113, E1881-E1889. doi: 10.1073/pnas.1602532113

Langmead, B., and Salzberg, S. L. (2012). Fast gapped-read alignment with Bowtie 2. Nat. Methods 9, 357-359. doi: 10.1038/nmeth.1923

Lau, P., Bossers, K., Janky, R., Salta, E., Frigerio, C. S., Barbash, S., et al. (2013). Alteration of the microRNA network during the progression of Alzheimer's disease. EMBO Mol. Med. 5, 1613-1634. doi: 10.1002/emmm.201201974

Leuba, G., Walzer, C., Vernay, A., Carnal, B., Kraftsik, R., Piotton, F., et al. (2008). Postsynaptic density protein PSD-95 expression in Alzheimer's disease and okadaic acid induced neuritic retraction. Neurobiol. Dis. 30, 408-419. doi: 10.1016/j.nbd.2008.02.012
Liu, C. G., Wang, J. L., Li, L., Xue, L. X., Zhang, Y. Q., and Wang, P. C. (2014). MicroRNA-135a and -200b, potential Biomarkers for Alzheimers disease, regulate $\beta$ secretase and amyloid precursor protein. Brain Res. 1583, 55-64. doi: 10.1016/j.brainres.2014.04.026

Love, S., Siew, L. K., Dawbarn, D., Wilcock, G. K., Ben-Shlomo, Y., and Allen, S. J. (2006). Premorbid effects of APOE on synaptic proteins in human temporal neocortex. Neurobiol. Aging 27, 797-803. doi: 10.1016/j.neurobiolaging.2005. 04.008

Luo, H., Wu, Q., Ye, X., Xiong, Y., Zhu, J., Xu, J., et al. (2014). Genome-wide analysis of miRNA signature in the APPswe/PS1DeltaE9 mouse model of alzheimer's disease. PLoS One 9:e101725. doi: 10.1371/journal.pone.0101725

Martinez, B., and Peplow, P. V. (2016). Blood microRNAs as potential diagnostic and prognostic markers in cerebral ischemic injury. Neural Regen. Res. 11, 1375-1378. doi: 10.4103/1673-5374. 191196

Muddashetty, R. S., Nalavadi, V. C., Gross, C., Yao, X., Xing, L., Laur, O., et al. (2011). Reversible inhibition of PSD-95 mRNA translation by miR-125a, FMRP phosphorylation, and mGluR signaling. Mol. Cell 42, 673-688. doi: 10.1016/j. molcel.2011.05.006

Niethammer, M., Kim, E., and Sheng, M. (1996). Interaction between the C terminus of NMDA receptor subunits and multiple members of the PSD-95 family of membrane-associated guanylate kinases. J. Neurosci. 16, 2157-2163.

Oakley, H., Cole, S. L., Logan, S., Maus, E., Shao, P., Craft, J., et al. (2006). Intraneuronal $\beta$-amyloid aggregates, neurodegeneration and neuron loss in transgenic mice with five familial Alzheimer's disease mutations: potential factors in amyloid plaque formation. J. Neurosci. 26, 10129-10140. doi: 10.1523/JNEUROSCI.1202-06.2006

O'Brien, R. J., and Wong, P. C. (2011). Amyloid precursor protein processing and Alzheimer's disease. Annu. Rev. Neurosci. 34, 185-204. doi: 10.1146/annurevneuro-061010-113613

Pozueta, J., Lefort, R., and Shelanski, M. L. (2013). Synaptic changes in Alzheimer's disease and its models. Neuroscience 251, 51-65. doi: 10.1016/j.neuroscience. 2012.05.050

Proctor, D. T., Coulson, E. J., and Dodd, P. R. (2010). Reduction in post-synaptic scaffolding PSD-95 and SAP-102 protein levels in the Alzheimer inferior temporal cortex is correlated with disease pathology. J. Alzheimers Dis. 21, 795-811. doi: 10.3233/JAD-2010-100090

Qiu, L., Tan, E. K., and Zeng, L. (2015). microRNAs and neurodegenerative diseases. Adv. Exp. Med. Biol. 888, 85-105. doi: 10.1007/978-3-319-22671-2_6

Reddy, P. H., Tripathi, R., Troung, Q., Tirumala, K., Reddy, T. P., Anekonda, V., et al. (2012). Abnormal mitochondrial dynamics and synaptic degeneration as early events in Alzheimer's disease: implications to mitochondria-targeted antioxidant therapeutics. Biochim. Biophy. Acta 1822, 639-649. doi: 10.1016/j. bbadis.2011.10.011

Reddy, P. H., Williams, J., Smith, F., Bhatti, J. S., Kumar, S., Vijayan, M., et al. (2017). MicroRNAs, aging, cellular senescence, and Alzheimer's disease. Prog. Mol. Biol. Transl. Sci. 146, 127-171. doi: 10.1016/bs.pmbts.2016.12.009

Saba, R., and Schratt, G. M. (2010). MicroRNAs in neuronal development, function and dysfunction. Brain Res. 1338, 3-13. doi: 10.1016/j.brainres. 2010.03.107

Salta, E., and De Strooper, B. (2012). Non-coding RNAs with essential roles in neurodegenerative disorders. Lancet Neurol. 11, 189-200. doi: 10.1016/S14744422(11)70286-1

Santa-Maria, I., Alaniz, M. E., Renwick, N., Cela, C., Fulga, T. A., Van Vactor, D., et al. (2015). Dysregulation of microRNA-219 promotes neurodegeneration through post-transcriptional regulation of tau. J. Clin. Invest. 125, 681-686. doi: 10.1172/JCI78421

Shao, N. Y., Hu, H. Y., Yan, Z., Xu, Y., Hu, H., Menzel, C., et al. (2010). Comprehensive survey of human brain microRNA by deep sequencing. BMC Genomics 11:409. doi: 10.1186/1471-2164-11-409

Sheng, M., Sabatini, B. L., and Südhof, T. C. (2012). Synapses and Alzheimer's disease. Cold Spring Harb. Perspect. Biol. 4:a005777. doi: 10.1101/cshperspect. a005777

Shipley, M. M., Mangold, C. A., and Szpara, M. L. (2016). Differentiation of the SH-SY5Y human neuroblastoma cell line. J. Vis. Exp. 108:53193. doi: $10.3791 / 53193$

Singh, A. K., Bissoyi, A., Kashyap, M. P., Patra, P. K., and Rizvi, S. I. (2017). Autophagy activation alleviates amyloid- $\beta$-induced oxidative 
stress, apoptosis and neurotoxicity in human neuroblastoma SH-SY5Y cells. Neurotox. Res. doi: 10.1007/s12640-017-9746-5 [Epub ahead of print].

Subramanian, A., Tamayo, P., Mootha, V. K., Mukherjee, S., Ebert, B. L., Gillette, M. A., et al. (2005). Gene set enrichment analysis: a knowledge-based approach for interpreting genome-wide expression profiles. Proc. Natl. Acad. Sci. U S A 102, 15545-15550. doi: 10.1073/pnas.0506580102

Tan, L., Yu, J. T., Tan, M. S., Liu, Q. Y., Wang, H. F., Zhang, W., et al. (2014). Genome-wide serum microRNA expression profiling identifies serum biomarkers for Alzheimer's disease. J. Alzheimers Dis. 40, 1017-1027. doi: 10.3233/JAD-132144

Wu, Q., Ye, X., Xiong, Y., Zhu, H., Miao, J., Zhang, W., et al. (2016). The protective role of microRNA-200c in Alzheimer's disease pathologies is induced by $\beta$ amyloid-triggered endoplasmic reticulum stress. Front. Mol. Neurosci. 9:140. doi: 10.3389/fnmol.2016.00140

Xun, Z., Lee, D. Y., Lim, J., Canaria, C. A., Barnebey, A., Yanonne, S. M., et al. (2012). Retinoic acid-induced differentiation increases the rate of oxygen consumption and enhances the spare respiratory capacity of mitochondria in SH-SY5Y cells. Mech. Ageing Dev. 133, 176-185. doi: 10.1016/j.mad. 2012.01.008

Zhang, J., Vinuela, A., Neely, M. H., Hallett, P. J., Grant, S. G., Miller, G. M., et al. (2007). Inhibition of the dopamine D1 receptor signaling by PSD-95. J. Biol. Chem. 282, 15778-15789. doi: 10.1074/jbc.M611485200

Conflict of Interest Statement: The authors declare that the research was conducted in the absence of any commercial or financial relationships that could be construed as a potential conflict of interest.

Copyright (C) 2017 Song and Kim. This is an open-access article distributed under the terms of the Creative Commons Attribution License (CC BY). The use, distribution or reproduction in other forums is permitted, provided the original author(s) or licensor are credited and that the original publication in this journal is cited, in accordance with accepted academic practice. No use, distribution or reproduction is permitted which does not comply with these terms. 